\title{
Development of micro-channel plates on a basis of aluminium oxide
}

\author{
Gleb Drobychev ${ }^{\mathrm{a}^{*}}$, Andrei Barysevich ${ }^{\mathrm{a}}$, Kirill Delendik ${ }^{\mathrm{b}}$, \\ Anton Karneyeu ${ }^{\mathrm{a}}$, Patrick Nédélec ${ }^{\mathrm{c}}$, Daniel Sillou ${ }^{\mathrm{c}}$, OlgaVoitik \\ ${ }^{a}$ Institute for Nuclear Problems, Minsk \\ ${ }^{b}$ Institute of Electronics, Minsk \\ ${ }^{c}$ Laboratory d'Annecy-le-Vieux de Physique des Particules
}

\begin{abstract}
Microchannel plates (MCP) are widely used in different fields of science and technology. They have good operational parameters, such as short response time $(<1 \mathrm{~ns})$, good spatial resolution, sensitivity up to single electron mode, high radiation hardness, immunity to magnetic fields. We propose a new kind of MCP based on an anodic aluminum oxide substrate formed by electrochemical oxidation of aluminium. This work presents preliminary results on $\mathrm{Al}_{2} \mathrm{O}_{3}$ based $\mathrm{MCPs}$. A research program is proposed which includes studies of the electrophysical properties of this MCPs in function of various geometric parameters (thickness, channel diameter to length ratio, effective surface, etc.).
\end{abstract}

Keywords: microchannel plate, electron amplification

PACS: 07.90.+c, 29.40.-n

\section{Introduction}

Electron Multipliers (EM) have a wide range of applications due to their good characteristics such as: high gain (10 3 $10^{6}$ ) and good operational stability. Nevertheless conventional EM with discrete dynodes present also some disadvantages such as: relatively poor time and spatial resolution, sensitivity to magnetic field and relatively large size.

Microchannel plates (MCP) consist of a matrix of parallel microchannels. Each one is a continuous dynode structure presenting the required resistive and secondary-emissive properties. Voltage is applied onto opposite surfaces of the plate. Incidence of primary electrons with channel walls cause an electron avalanche [1,2].

Modern MCPs are mostly produced from lead silicate glass (LSG). Their technology of fabrication is based on multiple processes of dragging and agglomeration of fibers having soluble metal or glass core resulting in production of primary multiple fibers. Agglomeration of multiple fiber bundles into MCP block is performed at high temperature. Afterwards the block is cut under certain angle into separate plates the surfaces of which are polished. MCP channels have hexagonal packaging, channel axis tilt is $5^{\circ}-15^{\circ}$ with respect to the perpendicular to MCP surface in order to diminish parasitic ion feedback. Conducting electrodes (chrome, nickel etc.) are sputtered on the polished surfaces. Transparency coefficient (ratio of the total area of input channel holes to the total MCP area) is about $50-70 \%$. Modern MCPs have a 0.4 - $4 \mathrm{~mm}$ thickness, and channels diameter $10-100 \mu \mathrm{m}$, channel-to-channel distance $6 \mu \mathrm{m}$, resistance is about $10^{8} \mathrm{Ohm}$. MCP channel length-to-diameter ratio (so called "operational aspect ratio" OAR) is in the range of $40-$ 100. They have high gain $\left(10^{5}-10^{6}\right)$; high sensitivity up to single electron mode; time resolution $<10^{-10} \mathrm{~s}$, high spatial resolution; ability to work in strong magnetic fields.

However, LSG MCP have serious disadvantages mostly bound to their fabrication process:

* Corresponding author. Tel.: +375-17-226 42 21; fax: +375 1722651 24; e-mail: Gleb.Drobychev@cern.ch. 
- Complex, labour consuming and expensive production technology;

- Large parameter deviation and low reproducibility of MCP characteristics due to glass irregularities;

- Image spottiness due to difference of thermal histories of MCP block elements;

- Presence of defects due to plate deformations caused by changes of glass composition and its temperature expansion coefficient;

- Operating in strong radiation environment causes about 10 - 15\% decrease of gain;

- Channels with diameters less than $10 \mu \mathrm{m}$ are impossible to produce with conventional methods which limits MCP spatial resolution;

- Large area MCP are difficult to produce

The development of MCP based on alternative technology, less expensive and more flexible could solve some of these problems. Many attempts to create new MCP technology were made during the last 20 years. Different microchannel structures made of glass, ceramics and other materials, and methods of conductive and secondary emissive layers creation are proposed. But in spite of an abundance of publications, a new MCP technology, able to concurrence the existing one did not emerge.

\section{Anodic aluminium oxide as a material for MCP production}

Recently, anodic aluminium oxide (AAO) was proposed as a possible option for MCP production. AAO is a dielectric material consisting of parallel, hexagonal packed cells, perpendicular to the surface of aluminium substrate. Each cell has an axial pore, closed by an oxide layer barrier on the side of the aluminium substrate. The cell diameter is mainly a function of the anodization voltage. Diameter of the pores depends on the nature of the electrolyte, and on several parameters of the anodization process. The adjustment of these parameters allows to adjust the dimensions of the cells and pores. Also a selective etching of cell walls can enlarge pore diameter [3, 4].

The technology of AAO production, including a deposition of oxide films with suitable electrophysical properties on the walls of the MCP channels was developed [5 - 7]. A deposition of these films modifies the conduction and secondary electron emission properties of the AAO MCP.

In comparison with conventional technology, the proposed one have several advantages:

- The use of standard microelectronics technology at all stages of MCP production (from plates growth up to production of operational MCP) will lower every costly aspect of the production process.

- The formation of microchannel structure by batch technology (instead of individual treatment of fibers), allow to raise MCP production yield and provide good reproducibility of parameters;

- The possibility to produce MCP with channel density up to $10^{10} \mathrm{~cm}^{-2}$, channels diameters from $10 \mathrm{~nm}$ up to several micrometers, and aspect ratio up to 600 allows to choose optimal combination of parameters and gain higher spatial resolution of AAO MCP compare to conventional LSG MCP;

- The possibility to produce of MCP with thickness from 40 to $200 \mu \mathrm{m}$ and up to $500 \mu \mathrm{m}$ with use of special technological methods,

- Ratio between surface of channels and surface of plate can be up to $50 \%$ without significant loss of mechanical hardness.

- Possibility to produce large area MCP up to $50 \times 50 \mathrm{~mm}$ and probably even up to $80 \times 80 \mathrm{~cm}$.

- Working temperature from -200 to $+500{ }^{\circ} \mathrm{C}$;

- Low sensitivity to magnetic fields;

- Extremely high radiation hardness.

- Possibility to implant by special technology a significant quantity of lead atoms into the MCP structure in order to increase its stopping power;

Listed parameters and characteristics of AAO MCP are impossible to obtain in LSG MCP produced by conventional methods. These advantages noticeably extend the field of application of microchannel multipliers. Industrial implementation of such technology will allow to decrease labour and material consumption and costs in dozens of times.

One can expect that microchannel electron multipliers on the base of anodic aluminum oxide are able to replace conventional MCPs in all fields of their current application, as well as in other fields where application of conventional MCPs is now limited by shortcomings of the existing technology.

\section{Simulation of the AAO MCP detection efficiency}

As well single AAO MCP have very small detection efficiency for the gamma with energies higher than $100 \mathrm{keV}$ a calorimeter for registration of gamma radiation with energy up to $1 \mathrm{MeV}$ should present a special design (see, for 
example, ref. $[8,9])$. A series of GEANT4 simulations were carried out to estimate a detection efficiency of single MCP as well as sandwiches of three MCP and absorbers as shown in Fig. 1.

The results of simulations are presented in Fig. 2 a, b and in Fig. 3 a, b.

As one can see that single MCP is efficient for electrons and X-rays registration, but inefficient for registration of gamma with energy higher than $30 \mathrm{keV}$. Use of sandwich design give an advantage. Among simulated beryllium looks the best for the energy region from 0 to $70 \mathrm{keV}$ and copper is preferable for region after $50 \mathrm{keV}$.

\section{Research Programme}

The research programme with the purposes to complete a development a production technology of AAO MCP with optimal electrophysical properties and to develop a prototype of calorimeter for registration of gamma radiation with energy up to $1 \mathrm{MeV}$ is already started. The following tasks are planned:

- $\quad$ To develop a technology of deposition of regular homogenious conductive and emissive coatings of with controllable electrophysical properties and good adhesion to the walls of deep narrow channels.

- $\quad$ To produce series of MCPs prototypes with different thickness, aspect ratio and size, and supplementary secondary emissive coatings (if the necessity of them will be found).

- To make regular research of MCP with various parameters in order to find their optimal combinations, which will provide the best electrophysical characteristics of MCP for different particular applications. The MCP amplification coefficient versus voltage, timing response (jitter), and MCP response to electrons excitation will be measured for single MCPs and sandwich on a basis of MCPs.

- $\quad$ To develop technology means to implant significant quantity of lead atoms into the MCP structure in order to increase MCP stopping power.

\section{First results of AAO MCP tests}

For the time being several different types of MCP with natural porosity are produced. They are of two thicknesses 40 and $190 \mu \mathrm{m}$ and have channels of $70-100 \mathrm{~nm}$ diameter. Some MCPs of $190 \mathrm{~nm}$ thickness were treated in order to increase channel diameter to $8 \mu \mathrm{m}$. The resistance was measured for all MCPs. Depending of MCP thickness it varies from tens to hundreds of MOhm.

Voltage-current characteristic of $40 \mu \mathrm{m}$ MCP with $70 \mathrm{~nm}$ channels diameter is shown in Fig. 4

\section{Acknowledgments}

The authors wish to express their gratitude to the French Ministery of Foreign Affairs for this project could be discussed and started thanks to the support of an ECONET program granted by the Ministery.

\section{References}

[1] J. L. Wiza. Nucl. Instr. Meth., 162 (1979) 587.

[2] P.M. Shikhaliev. Rev. Sci. Instrum., Vol. 67, No. 3, March 1996

[3] O. L. Voitik, K. I. Delendik. Proceeding of SPIE 2001 Int. Symp. on Microelectronics and Micro-Electro-Mechanical Systems (MICRO/MEMS 2001). 17-19 December 2001, Adelaide, Australia, 7 pp.

[4] Delendik K. I., Voitik O. L. Proceeding of Fourth Int. Workshop on High-Aspect-Ratio Micro-Structure Technology. June 2001. Baden-Baden, Germany. 2pp.

[5] A.S. Kurilin, et. al. Appl. Surf. Sci. 111 (1997) p.295-301

[6] A.S. Kurilin, et al. Nucl. Instr. and Meth. A 419 (1998) 667.

[7] Emeliantchik I.F., Rumiantsau V.S. et.al. in: Selected scientific papers of BSU, Minsk, 2001, p. 303-322.

[8] P. M. Shikhaliev, Rev. Sci. Instr. 68, (1997) 10.

[9] P.M. Shikhaliev. Nucl. Instr. and Meth. in Phys. Res. A, 379 (1996) 307. 


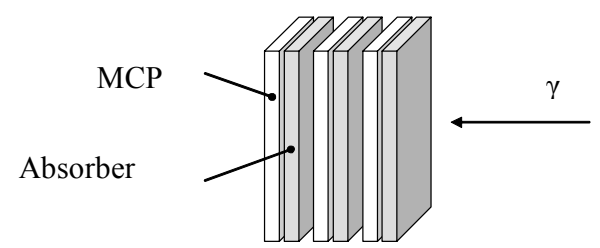

Fig. 1. Sandwich of AAO MCP $(100 \mu \mathrm{m})$ and $500 \mu \mathrm{m}$ absorber.
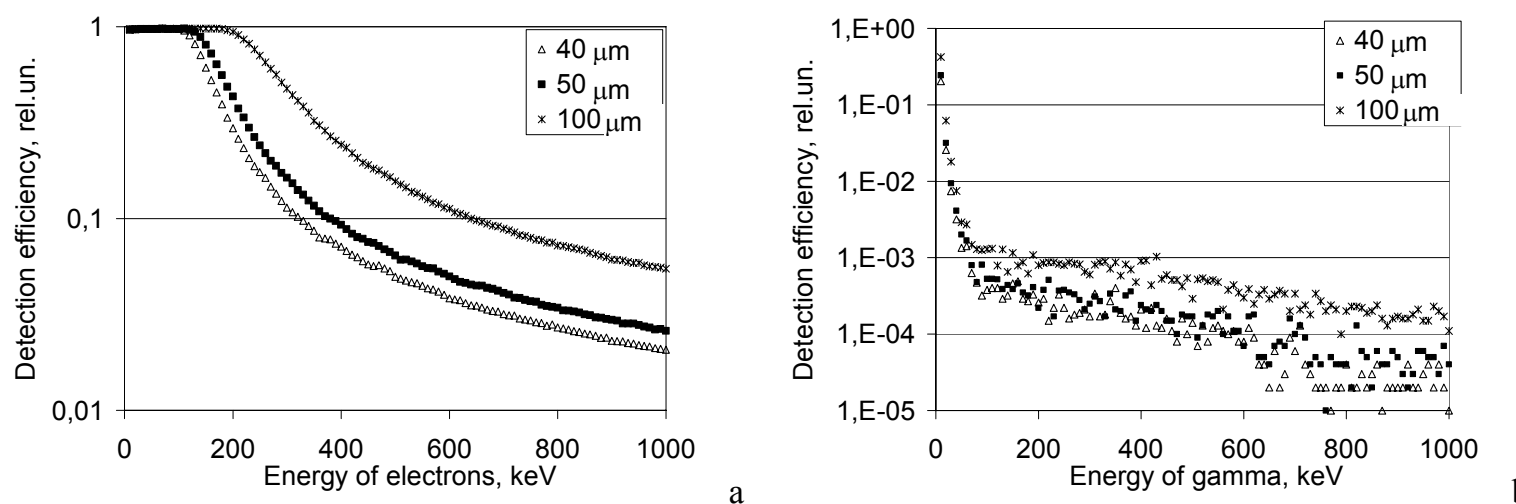

Fig. 2. Detection efficiency for electrons (a) and gamma (b) for single AAO MCP of different thickness.
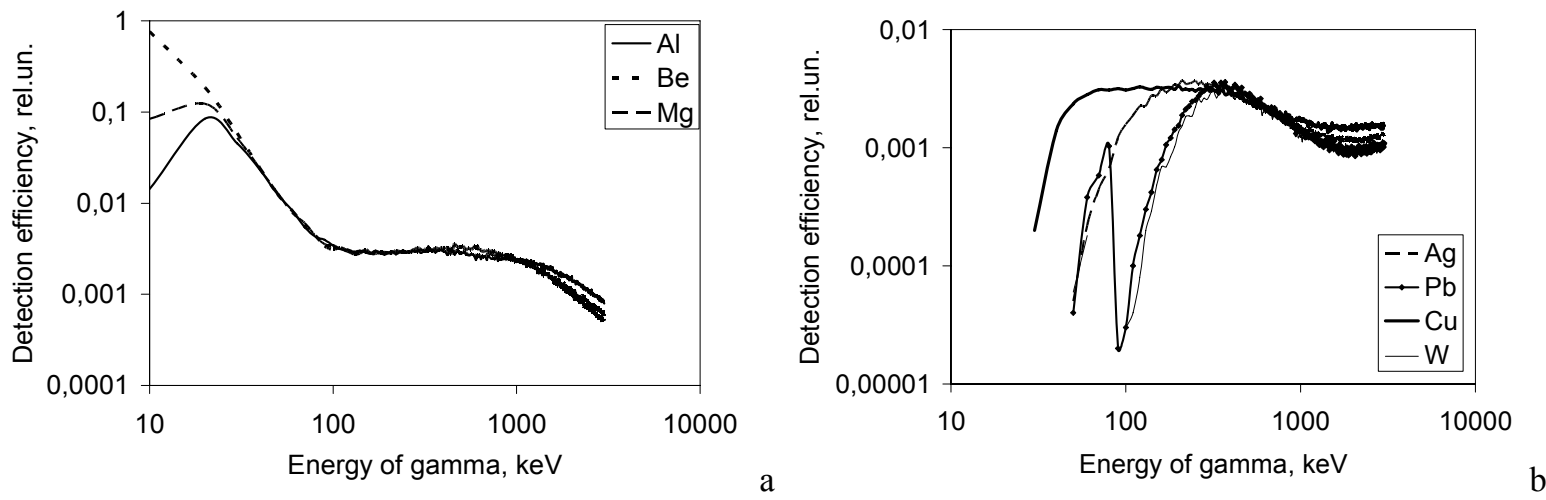

Fig. 3. Detection efficiency of sandwich of AAO MCP and different metal absorbers.

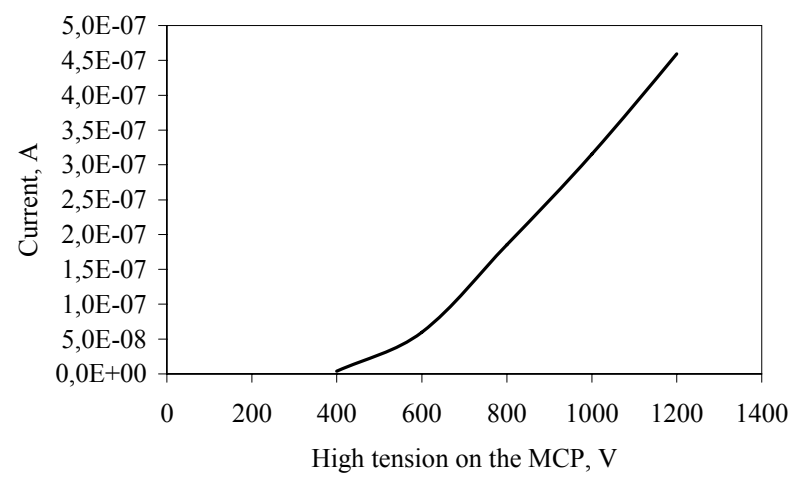

Fig. 4. Voltage-current characteristic of $40 \mu \mathrm{m} \mathrm{MCP}$ with $70 \mathrm{~nm}$ channels diameter. 